EPJ Web of Conferences 73, 07009 (2014)

DOI: $10.1051 /$ epjconf/20147307009

(C) Owned by the authors, published by EDP Sciences, 2014

\title{
Reactions with pions and vector mesons in the sector of odd intrinsic parity
}

\author{
Carla Terschlüsen ${ }^{\mathrm{a}}$, Bruno Strandberg and Stefan Leupold \\ Department of Physics and Astronomy, Uppsala University, Sweden
}

\begin{abstract}
In chiral perturbation theory, the leading-order contribution to reactions with pions in the sector of odd intrinsic parity is defined by the Wess-Zumino-Witten structure. This structure is supplemented by a simple vector-meson Lagrangian where the vector mesons are described by antisymmetric tensor fields. With the rho-omega-pion coupling as the only parameter in the sector of odd intrinsic parity, i.e. without additional contact terms, one can achieve a proper description of the pion transition form factor and the three-pion production in electron-positron collisions.
\end{abstract}

\section{Introduction}

One of the challenges in hadron physics is the description of the low-energy regime. Due to the running coupling constant in QCD, perturbation theory can only be used for high energies but not for low energies. Instead, this energy regime can be described using effective theories with hadrons instead of quarks and gluons as relevant degrees of freedom. An effective theory for the lowest energy regime is chiral perturbation theory $(\chi \mathrm{PT})$ which uses the low-lying pseudoscalar octet (pions, kaons, $\eta)$ as relevant degrees of freedom [1]. In $\chi \mathrm{PT}$, all vector mesons are treated as heavy. Hence, it is not applicable for the energy range of the hadronic resonances $\left(\rho, \omega, K^{*}, \phi, \eta^{\prime}\right)$.

Although there exist successful phenomenological models as, e.g., vector meson dominance to describe the energy regime of hadronic resonances, it is desirable to develop effective theories or alternative systematic approaches for this energy regime. Furthermore, the transition between $\chi \mathrm{PT}$ and such theories for higher energies is of interest. Here, the transition between the energy regime where $\chi \mathrm{PT}$ is applicable and higher energies will be explored restricted to the sector of odd intrinsic parity and $\mathrm{SU}(2)$, i.e. only considering the pseudoscalar pions and the $\rho$ and $\omega$ vector mesons. For more details, see [2].

Mesons with spin $J$ and parity $P$ have an intrinsic parity of $P \cdot(-1)^{J}$. Therewith, vector mesons have even and pseudoscalar mesons odd intrinsic parity. Thus, in $\mathrm{SU}(2)-\chi \mathrm{PT}$ strong or electromagnetic reactions in the sector of odd intrinsic parity correspond to reactions with an odd number of pions. Such reactions are governed by the Wess-Zumino-Witten (WZW) action. In leading order, the relevant terms

\footnotetext{
ae-mail: carla.terschluesen@physics.uu.se
}

This is an Open Access article distributed under the terms of the Creative Commons Attribution License 4.0, which permits unrestricted use, distribution, and reproduction in any medium, provided the original work is properly cited. 
for $\mathrm{SU}(2)$ are given by [3]

$$
\mathcal{L}_{\mathrm{WZW}}=-\frac{n e^{2}}{24 \pi^{2} f_{\pi}} \varepsilon^{\mu \nu \alpha \beta} \partial_{\mu} \pi^{0} \partial_{\nu} \mathcal{A}_{\alpha} \mathcal{A}_{\beta}+i \frac{n e}{24 \pi^{2} f_{\pi}^{3}} \partial_{\mu} \pi^{+} \partial_{\nu} \pi^{-} \partial_{\alpha} \pi^{0} \mathcal{A}_{\beta}
$$

where $\mathcal{A}$ denotes photon fields. Due to topological arguments [3], the parameter $n$ is equal to the number of colors, $n=N_{c}=3$, and the WZW Lagrangian is parameter independent ${ }^{1}$.

The low-energy constants in $\chi \mathrm{PT}$ are saturated by vector-meson exchange [1]. Therefore, $\chi \mathrm{PT}$ at next-to-leading order is equivalent to a Lagrangian with both pseudoscalar and vector mesons. Relevant here is the following part of this vector-meson Lagrangian in the sector of even intrinsic parity,

$$
\mathcal{L}_{\text {vec }}^{\text {even }}=-\frac{e f_{V}}{3}\left(3 \rho_{\mu \nu}^{0}+\omega_{\mu \nu}\right) \partial^{\mu} \mathcal{A}^{\nu}+i \frac{f_{V} h_{P}}{8 f_{\pi}^{2}} \operatorname{tr}\left(V_{\mu \nu} \partial^{\mu} \Phi \partial^{v} \Phi\right)
$$

with the pion-decay constant $f_{\pi}=90 \mathrm{MeV}, V_{\mu \nu}$ denoting the vector meson fields given in antisymmetric tensor representation and $\Phi$ the pion fields,

$$
V_{\mu \nu}=\left(\begin{array}{cc}
\rho_{\mu \nu}^{0}+\omega_{\mu \nu} & \sqrt{2} \rho_{\mu \nu}^{+} \\
\sqrt{2} \rho_{\mu \nu}^{-} & -\rho_{\mu \nu}^{0}+\omega_{\mu \nu}
\end{array}\right), \quad \Phi=\left(\begin{array}{cc}
\pi^{0} & \sqrt{2} \pi^{+} \\
\sqrt{2} \pi^{-} & -\pi^{0}
\end{array}\right) .
$$

In the sector of odd intrinsic parity, we supplement 2 by

$$
\mathcal{L}_{\mathrm{vec}}^{\text {odd }}=-\frac{h_{A}}{8 f_{\pi}} \varepsilon^{\mu \nu \alpha \beta} \operatorname{tr}\left(\left\{V_{\mu \nu}, \partial^{\tau} V_{\tau \alpha}\right\} \partial_{\beta} \Phi\right) .
$$

The parameters $f_{V}, h_{P}$ and $h_{A}$ still have to be determined.

In $\chi \mathrm{PT}$, all vector mesons are treated as heavy. For momenta $p$ much smaller than the vector meson mass $m_{V}$, the WZW Lagrangian $\mathcal{L}_{\text {WZW }}$ would therefore be of order $p^{4}$ whereas the vector-meson Lagrangian $\mathcal{L}_{\text {vec }}^{\text {odd }}$ of order $p^{6}$. On the other hand, for momenta $p \approx m_{V} \mathcal{L}_{\text {WZW would still be of order }}$ $p^{4}$ but $\mathcal{L}_{\text {vec }}^{\text {odd }}$ of order $p^{2}$ according to the counting scheme of [4] where both pseudoscalar and vector mesons are treated as light. Therefore, both Lagrangians are formally never of the same order. Hence, there exist three possibilities to calculate reactions with an odd number of pions: First, only the pure $\chi$ PT contribution $\mathcal{L}_{\text {WZW }}$ could be used. Second, only the vector-meson contribution $\mathcal{L}_{\text {vec }}^{\text {odd }}$ could be used. Third, as a more phenomenological approach both Lagrangians could be used $^{2}$. In the following it will be shown that one indeed needs both Lagrangians to be able to describe the available experimental data.

\section{Determination of open parameters}

In the sector of even intrinsic parity, the reaction $e^{+} e^{-} \rightarrow \pi^{+} \pi^{-}$and the pion form factor can be used to determine the open parameters $f_{V}$ and $h_{P}$ in (2). The scattering reaction can happen both with a direct $2 \pi-\gamma$ coupling given by usual $\chi \mathrm{PT}$ or via an intermediate $\rho^{0}$ meson described with (2). Including pion-pion rescattering, the best agreement with the available experimental data for the pion form factor is given for $f_{V}=150 \mathrm{MeV}$ and $h_{P}=1.64$ (see left-hand side in Fig. 1 and [2] for more details).

In the vector meson sector, the only open parameter for odd intrinsic parity is $h_{A}$. To determine $h_{A}$, the decay $\omega \rightarrow \pi^{+} \pi^{-} \pi^{0}$ including pion-pion rescattering can be used. However, comparison to experimental data gives only access to the absolute value, $\left|h_{A}\right|=2.02$. The sign of $h_{A}$ is not a matter of convention since the sign of the parameter $n$ in the WZW Lagrangian 1 was already chosen before. The sign of $h_{A}$ relative to $\mathcal{L}_{\text {WZW }}$ can be determined by considering the reaction $e^{-} \gamma \rightarrow e^{-} \pi^{0}$. Again, this reaction can happen directly described by $\chi \mathrm{PT}$ including the parameter $n$ or via virtual $\omega$ and $\rho^{0}$ mesons

\footnotetext{
${ }^{1}$ Note that the sign of $n$ is pure convention corresponding to the field $+\pi$ producing pions instead of $-\pi$.

2 Note that this is no full next-to-leading-order calculation since the vector-meson Lagrangian is only given in leading order.
} 

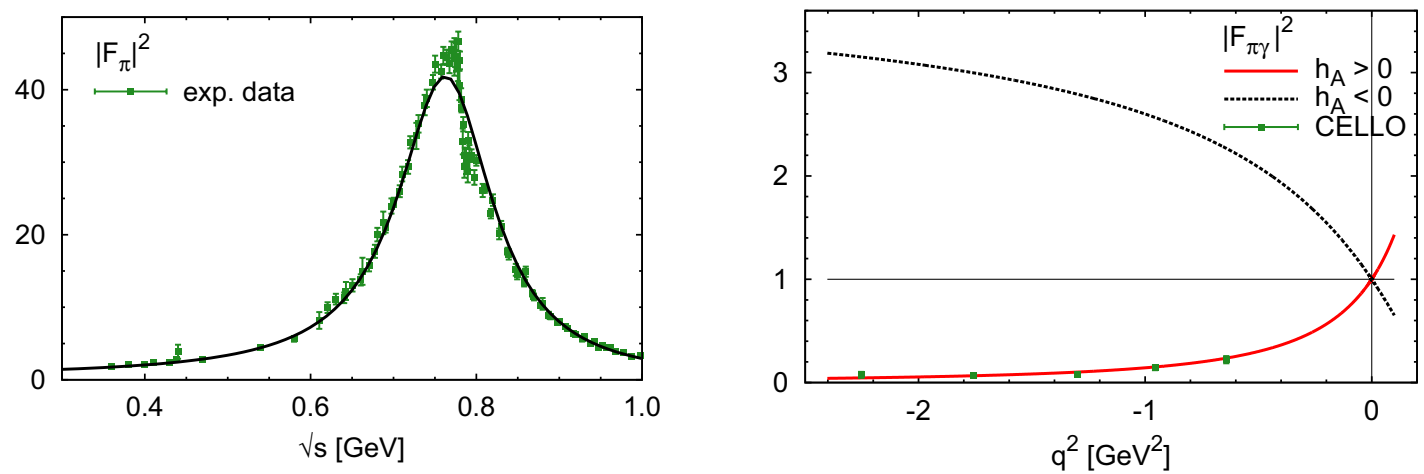

Figure 1. Left: pion form factor compared to experimental data [5-7]. Right: normalized $\pi^{0}-\gamma$ transition form factor for $h_{A}>0$ (red solid line) and for $h_{A}<0$ (black dotted line) compared to data taken by the CELLO collaboration [8].

including the parameter $h_{A}$. Comparing the normalized $\pi^{0}-\gamma$ transition form factor,

$$
F_{\pi \gamma}\left(q^{2}=m_{e^{+} e^{-}}^{2}\right)=1+\frac{4 \pi^{2} f_{V}^{2}}{n m_{\rho / \omega}^{2}} \frac{q^{2}}{m_{\rho / \omega}^{2}-q^{2}} h_{A},
$$

with an averaged $\rho$ - $\omega$ mass $m_{\rho / \omega}$ for both positive and negative $h_{A}$ to experimental data yields $h_{A}>0$ (see right-hand side in Fig. 1). Note that the absolute value of the parameter has not been fitted to the data. Using only $\mathcal{L}_{\mathrm{WZW}}\left(h_{A}=0\right)$ would yield $F \equiv 1$, using only the vector-meson Lagrangian $\mathcal{L}_{\text {vec }}=\mathcal{L}_{\text {vec }}^{\text {even }}+\mathcal{L}_{\text {vec }}^{\text {odd }}$ would not allow to normalize since the (unnormalized) form factor $f \sim q^{2}$. Thus, both Lagrangians are needed. Then one obtains a very good description of the experimental data.

\section{Applications}

The information obtained from the single-virtual form factor can be used to calculate the doublevirtual decay $\pi^{0} \rightarrow 2 e^{+} 2 e^{-}$. Since the measured momenta $\left(q_{1}, q_{3}\right)$ of the electrons and $\left(q_{2}, q_{4}\right)$ of the positrons can be produced in two different ways (see left-hand side in Fig. 2), the decay width for the double-virtual decay consists of two parts: A direct contribution $\Gamma_{\text {dir. }}$ depending only on two momenta combinations $q_{i}+q_{j}$ and an interference contribution $\Gamma_{\text {int. }}$ depending on all possible combinations $q_{i}+q_{j}$. It turns out that the interference contribution is less than $1 \%$ of the direct contribution yielding a very good agreement of the total width with the experimental value,

$$
\begin{aligned}
& \Gamma_{\text {dir. }}=2.70 \cdot 10^{-13} \mathrm{GeV}, \quad \Gamma_{\text {int. }}=-0.02 \cdot 10^{-13} \mathrm{GeV}, \\
& \Gamma_{\pi^{0} \rightarrow 2 e^{+} 2 e^{-}}=\Gamma_{\text {dir. }}+\Gamma_{\text {int. }}=2.68 \cdot 10^{-13} \mathrm{GeV}, \\
& \Gamma_{\pi^{0} \rightarrow 2 e^{+} 2 e^{-}}^{\text {exp. }}=(2.58 \pm 0.13) \cdot 10^{-13} \mathrm{GeV} .
\end{aligned}
$$

For the scattering process $e^{+} e^{-} \rightarrow \pi^{+} \pi^{-} \pi^{0}$, one gets a vector contribution including the decay $\omega^{*} \rightarrow \pi^{+} \pi^{-} \pi^{0}$ and a contribution from $\mathcal{L}_{\text {WZW }}$ with a direct $3 \pi-\gamma$ vertex. The plot on the right-hand side in Fig. 2 shows the calculated cross section for the vector contribution only (black dashed line) and for both contributions (red solid line) compared to experimental data for a total energy $\sqrt{s}$ between $0.6 \mathrm{GeV}$ and $1 \mathrm{GeV}$. Both calculations agree well with the experimental data whereby $\mathcal{L}_{\text {WZW }}$ influences the cross section only slightly. 

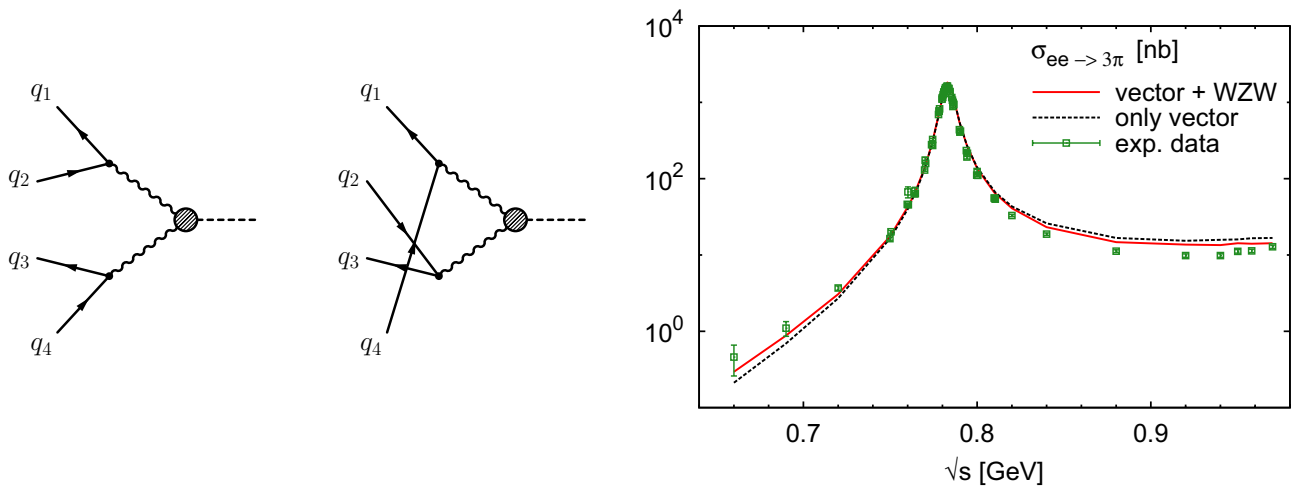

Figure 2. Left: possibilities to produce the measured momenta of electrons and positrons in the double-virtual pion decay. Right: cross section for the scattering reaction $e^{+} e^{-} \rightarrow 3 \pi$ calculated with both Lagrangians (red solid line) and with $\mathcal{L}_{\text {vec }}$ only (black dotted line) compared to experimental data $[9,10]$.

\section{Summary}

Instead of describing reactions with an odd number of pions with the leading $\chi \mathrm{PT}$ contribution, the Wess-Zumino-Witten Lagrangian, or only with a Lagrangian including vector meson, both Lagrangians were used. Thereby, the three open parameters $\left(f_{V}, h_{P},\left|h_{A}\right|\right)$ of the vector Lagrangian can be fixed from the decay $\omega \rightarrow 3 \pi$ and the pion form factor. The sign of the parameter $h_{A}$ relative to the Wess-ZuminoWitten Lagrangian can be fixed by comparing the $\pi^{0}-\gamma$ transition form factor to experimental data. A description of the experimental data is only possible if both Lagrangians are used. Furthermore, the reactions $\pi^{0} \rightarrow 2 e^{+} 2 e^{-}$and $e^{+} e^{-} \rightarrow 3 \pi$ can be described very well using the previously determined values for the parameters of the vector-meson Lagrangian.

\section{References}

[1] J. Gasser, H. Leutwyler, Ann. Phys. 158, 142 (1984)

[2] C. Terschlüsen, B. Strandberg, S. Leupold, F. Eichstädt, Eur. Phys. J. A 49, 116 (2013)

[3] E. Witten, Nucl. Phys. B 223, 422 (1983)

[4] C. Terschlüsen, S. Leupold, M.F.M. Lutz, Eur. Phys. J. A 48, 190 (2012)

[5] L. Barkov et al., Nucl. Phys. B 256, 365 (1985)

[6] R.R. Akhmetshin et al., Phys. Lett. B 527, 161 (2002)

[7] R.R. Akhmetshin et al., Phys. Lett. B 578, 285 (2004)

[8] H.J. Behrend et al., Z. Phys. C 49, 401 (1991)

[9] M.N. Achasov et al., Phys. Rev. D 68, 052006 (2003)

[10] R.R. Akhmetshin et al., Phys. Lett. B 476, 33 (2000) 